\title{
Norois
}

Environnement, aménagement, société

$240 \mid 2016$

Aménagement foncier, risque côtier, évolution des fleuves

\section{Les formations superficielles entre la Loire et le Loir (sud-ouest du Bassin parisien) : les enseignements de l'analyse sédimentologique}

Detritic deposits between the Loire and the Loir (Paris basin): sedimentological study

Jean-Pierre Larue et Édouard de Bélizal

\section{(2) OpenEdition}

\section{Journals}

\section{Édition électronique}

URL : http://journals.openedition.org/norois/5942

DOI : $10.4000 /$ norois. 5942

ISBN : 978-2-7535-5362-0

ISSN : $1760-8546$

\section{Éditeur}

Presses universitaires de Rennes

Édition imprimée

Date de publication : 30 novembre 2016

Pagination : 43-57

ISBN : 978-2-7535-5356-9

ISSN : 0029-182X

\section{Référence électronique}

Jean-Pierre Larue et Édouard de Bélizal, « Les formations superficielles entre la Loire et le Loir (sudouest du Bassin parisien) : les enseignements de l'analyse sédimentologique », Norois [En ligne], 240 2016, mis en ligne le 30 novembre 2018, consulté le 24 novembre 2020. URL : http:// journals.openedition.org/norois/5942 ; DOI : https://doi.org/10.4000/norois.5942 


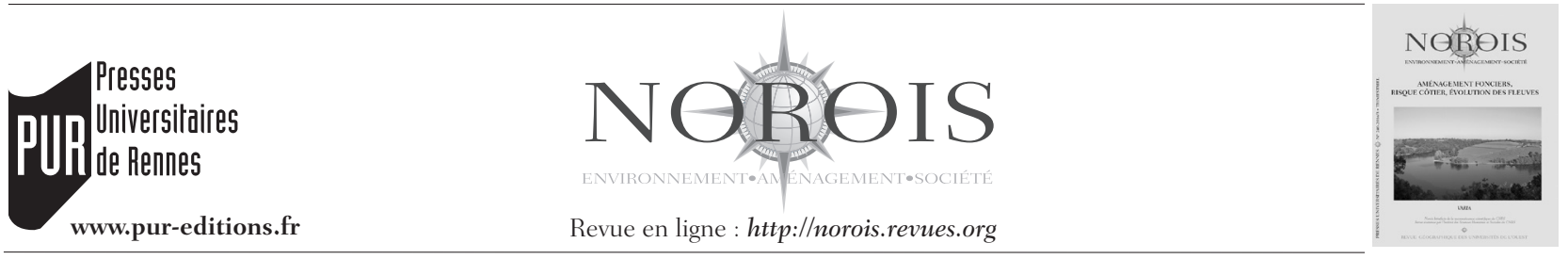

\title{
Les formations superficielles entre la Loire et le Loir (sud-ouest du Bassin parisien) : les enseignements de l'analyse sédimentologique
}

\author{
Detritic Deposits between the Loire River and the Loir River (Paris Basin): \\ Sedimentological Study
}

\author{
Jean-Pierre LARUE ${ }^{a}$ et Édouard DE BelizAL ${ }^{b}$
}

\begin{abstract}
a Professeur émérite, Département de Géographie, Faculté des Lettres et Sciences Humaines, Université de ParisEst-Créteil et CNRS, UMR 8591, Laboratoire de Géographie Physique (LGP), Meudon. (larue@u-pec.fr)

b CNRS, UMR 8591, Laboratoire de Géographie Physique (LGP), Meudon. (edouard.dbelizal@gmail.com)
\end{abstract}

Résumé : L’analyse sédimentologique des formations détritiques tertiaires et quaternaires situées sur l'interfluve Loire-Loir, en Touraine, permet de caractériser plusieurs nappes détritiques. Datée du Pliocène supérieur par la présence de grains de quartz issus de ponces du Mont-Dore, la formation des Sables de Château-Renault et de Montreuil n'est constituée pour les autres dépôts que d'apports locaux, ce qui va à l'encontre de l'hypothèse d'un écoulement chenalisé de la Loire-amont vers l'Atlantique à cette époque. Issus du remaniement de dépôts marins locaux, les Sables de Mazières sont probablement un peu plus récents. Les premières alluvions quaternaires au nord de Nazelles, mieux classées, très riches en zircon, témoignent d'un transport fluviatile lointain effectué par une paléo-Loire chenalisée. Enfin, des sables soufflés, provenant des alluvions du Val de Loire, recouvrent localement les Sables de Mazières.

\begin{abstract}
In the Touraine area, the plateaux which form the Loire-Loir interfluve stand at rather low and equal altitudes and bear locally detrital deposits which are not easy to identify (fig. 1,2). The sedimentological study of those deposits makes it possible to distinguish several formations (fig. 3, 4 and tables 1, 2): The Chateau-Renault sands and the Montreuil sands look very much the same because of their bad sizing, their strong weathering and their heavy minerals distribution: a great deal of staurolite and not any garnet. The presence of pumice quartz, coming from the Mont-Dore eruption, makes it likely to date the Montreuil sands back to the Upper Pliocene. The Mazières sands, located north-west of Tours, come from the erosion of local marine deposits as it is proved by the morphoscopy and the absence of garnet and zircon which are present in the Faluns and the Sologne sands. The alluvial deposits, located in the north of Nazelles, are better grain-sized and very rich in zircon and therefore may have been brought from upstream by the river Loire. The upper sands covering the Mazières sands are abundant in round and mat grains and heavy minerals of volcanic origin and have been brought by the wind from the alluvial deposits of the Val de Loire.

If a part of the Montreuil sands certainly dates back to the Upper Pliocene, on the other hand their sedimentological characteristics invalidate the hypothesis of a flow of the upstream Loire towards the Atlantique in that period. The curving towards the Atlantique did not take place until the ground was slightly abrased before the accumulation of the allwial deposits which are preserved north of Nazelles (fig. 5). Coming from local substratum, those detrital formations, which are preserved on very closed levels, prove the relative tectonic steadiness in the Touraine area.
\end{abstract}

Mots clés : alluvions - sédimentologie - minéraux lourds - paléogéographie - paleoenvironnement - Quaternaire

Keywords: alluvial deposit - sedimentology - heavy minerals - palaeogeography - paleoenvironment, Quaternary 


\section{INTRODUCTION}

En Touraine, les plateaux d'altitude modeste (90 à $180 \mathrm{~m}$ ) situés sur la rive droite de la Loire, et formant l'interfluve Loire-Loir (figure 1 - planche V) portent localement des formations détritiques tertiaires et quaternaires dont l'origine et l'âge précis restent hypothétiques. Ces « Sables et graviers continentaux » des auteurs des cartes géologiques (Alcaydé, 1968; Lorain et al., 1972; Manivit et Martins, 1974; Rasplus et al., 1974; Macaire et Desprez, 1977) sont présumés postérieurs aux Faluns helvétiens (Langhien et Serravalien), qu'ils ravinent, mais constituent-ils une nappe détritique unique? Si certains lambeaux sont issus d'une dissolution partielle ou totale des Faluns (Denizot, 1927), la plupart résultent d'un transport fluviatile, effectué pendant la régression post-helvétienne comme le pense C. Klein (1973 et 1990) ou au Pliocène supérieur comme le suggèrent C. Cavelier et al. (1993). Pour J.-J. Macaire (1981), la plus grande partie de ces dépôts résulterait des apports d'un pré-Cher. La réponse à ces questions apporterait des arguments pour trancher l'épineux problème des changements de cours de la Loire dans le Bassin parisien depuis le Miocène (Dewolf et Penven, 2007 ; Étienne et Larue, 2011).

Les objectifs de cette étude sont de mieux caractériser ces "Sables et graviers continentaux », afin de déterminer s'ils constituent une seule ou plusieurs nappes détritiques et d'en connaître l'origine, mais aussi de préciser leurs rapports avec les formations voisines plus anciennes et plus récentes et ainsi d'esquisser l'évolution paléogéographique de cette région. Nous avons choisi la rive droite de la Loire, car elle concentre les plus fortes variétés et densités de lambeaux détritiques, et présente de beaux affleurements à la faveur d'excavations d'origine diverse.

Les méthodes utilisées sont les analyses géomorphologique et sédimentologique. Afin d'identifier plusieurs formations au sein de ces dépôts dont l'épaisseur n'excède guère $3 \mathrm{~m}$, sauf à l'est de Château-Renault où elle atteint 10,20 m, les échantillons sableux prélevés (150 à 200 g environ par échantillon) ont subi les analyses sédimentologiques suivantes : tamisage et microgranulométrie laser, étude morphoscopique des sables, détermination des minéraux argileux et des minéraux lourds. Les
31 échantillons prélevés sur le terrain sont localisés sur la figure 2 et dans le tableau 1. Dix représentent les «Sables et graviers continentaux », notés Sables de Château-Renault, Sables de Montreuil et Sables de Mazières, quatre concernent les sables soufflés depuis le Val de Loire ( ${ }^{\circ} 2,3$, 6 et 7 ), les autres caractérisent les Faluns du Miocène moyen et supérieur $\left(\mathrm{n}^{\circ} 14,42\right.$ et 43$)$, les Sables de Sologne du Miocène moyen ( $\mathrm{n}^{\circ} 46,50,51,52$ et 53$)$, la très haute terrasse de la Loire ( $\mathrm{n}^{\circ} 9,18$ et 27 ), les autres terrasses de la Loire ( ${ }^{\circ}$ 23, 29, 31, 32 et 33) et les apports du Loir (n⿳3 32).

\section{DES FORMATIONS DÉTRITIQUES SITUÉES À DES ALTITUDES PEU DIFFÉRENTES}

Sur l'interfluve Loire-Loir, les « Sables et graviers continentaux » reposent sur une surface d'érosion représentant, soit une véritable pénéplaine selon G. Denizot (1927), soit une surface polygénique faite de la juxtaposition de différentes formations d'âges divers, une «surface en marqueterie " selon J.-C. Yvard (1978 et 1995). Cette surface recoupe des terrains d'âges variés : argiles à silex développées sur la craie crétacée, sables et cailloutis de l'Éocène inférieur et moyen, calcaires lacustres de l'Éocène supérieur, faluns dont l'âge s'étale selon les auteurs du Langhien au Tortonien, voire au Pliocène (André et al., 2003; Courville et Crônier, 2003). Ces derniers sont toutefois conservés en creux, dans de petites dépressions, comme à La Mossuère, au sud-ouest de Blois, ou dans d'anciennes rías, comme celle de la Brenne, à Château-Renault (figure 2). Les cours d'eau actuels s'encaissent de 40 à $60 \mathrm{~m}$ dans les plateaux qui forment une vaste gouttière centrée sur la Loire (Rasplus, 1978).

Malgré des variations locales non négligeables, les faciès de ces formations situées à des altitudes peu différentes (figure 1) apparaissent plutôt homogènes :

- au sud-ouest de Montreuil, à 110 m d'altitude, la petite excavation de La Chevallerie $\left(n^{\circ} 8\right.$, tableau 1) permet d'observer la formation des Sables de Montreuil. La coupe montre sur 3 m d'épaisseur des sables argileux ocre-rouille dont la structure chenalisante à stratifications obliques est soulignée par des lits de graviers de quartz et de silex. Ces dépôts de barres de chenal formés de lentilles à stratifica- 


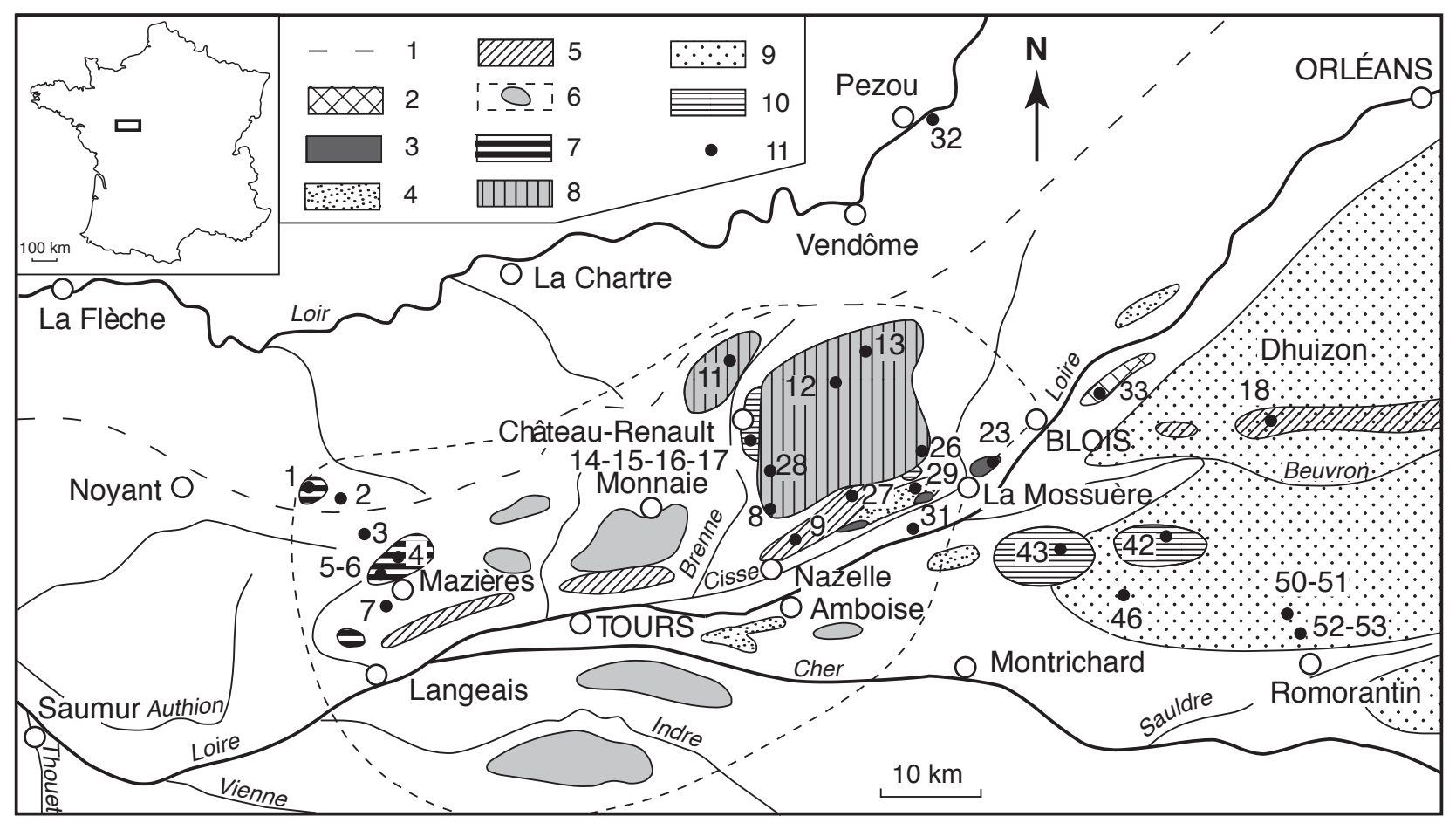

Figure 2 : Carte de localisation des dépôts détritiques

1 : ligne de partage des eaux actuelle entre la Loire et le Loir; 2 : nappe alluviale Fx de la Loire; 3 : nappe alluviale Fw de la Loire; 4 : nappe alluviale Fv de la Loire; 5 : nappe alluviale Fu de la Loire; 6 : principaux placages et limites de l'extension des « Sables et graviers continentaux » de Touraine; 7 : Sables de Mazières; 8 : Sables de Château-Renault et de Montreuil ; 9 : Sables de Sologne; 10 : Faluns de Touraine; 11 : échantillon analysé. L'extension des sables soufflés (échantillons 2, 3, 6 et 7) n'est pas représentée.

Location map

1: drainage divide; 2: Fx Loire alluvial deposits; 3: Fw Loire alluvial deposits; 4: Fv Loire alluvial deposits; 5: Fu Loire alluvial sheet; 6: oucrops and limits of the Touraine "Sables et graviers continentaux"; 7: Mazières sands; 8: Château-Renault and Montreuil sands; 9: Sologne sands; 10: Falun; 11: analysed samples. Limits of wind-brought sands (samples 2, 3, 6 and 7) are not drawned.

tions obliques tronquées à leur partie supérieure par des lits discontinus de graviers suggèrent un milieu de plaine alluviale à chenaux tressés (Miall, 1996). L'échantillon $n^{\circ} 8$ a été prélevé dans une lentille de sables fins (figure 2);

- au sud-ouest de Blois, à 104 m d'altitude, près de La Touche, toujours dans la formation des Sables de Montreuil, une petite excavation montre sur 4,50 m d'épaisseur des sables feldspathiques plus ou moins argileux (échantillon $n^{\circ} 26$ ) contenant des graviers de silex, de chailles de couleur noire et de quartz de 1 à $2 \mathrm{~cm}$ de diamètre ;

- au nord de Nazelles, à 105 m d'altitude, les fossés de la route de Reugny révèlent des sables argileux ocre-rouille à graviers de quartz de $2 \mathrm{~cm}$ de diamètre en moyenne ( $\left.n^{\circ} 9\right)$ qui ressemblent fortement aux précédents, mais qui appartiennent à la très haute terrasse de la Loire $(\mathrm{Fu})$;

- au sud-est de Château-Renault, la sablière de la Sablonnière permet d'observer au-dessus des Faluns $\left(n^{\circ} 14\right)$ la formation des Sables de Château-Renault. On peut observer des sables fins ocres $\left(n^{\circ} 15\right)$ épais de 1,50 m, puis des limons argileux gris $\left(n^{\circ} 16\right)$ de $1 \mathrm{~m}$ de puissance et au sommet, sur $1 \mathrm{~m}$ d'épaisseur, des sables grossiers ocre-rouille $\left(n^{\circ} 17\right)$ incluant des lits de silex de 5 à $7 \mathrm{~cm}$ de diamètre et quelques lentilles de sables fins. Situé à 115 m d'altitude, ce remblaiement n'atteint pas le sommet du plateau qui culmine à $147 \mathrm{~m}$ plus au sud-est;

- au nord-ouest de Tours, près de la forêt de Mazières, à 100 m d'altitude, la pose de poteaux électriques nous a permis d'observer la formation des Sables de Mazières représentée par des sables argileux ocre-rouille ( ${ }^{\circ} 4$ et $\left.n^{\circ} 5\right)$ sur 1 m d'épaisseur. Ils sont recouverts de sables gris peu argileux $\left(\mathrm{n}^{\circ} 6\right)$ de $50 \mathrm{~cm}$ de puissance qui appartiennent aux sables soufflés selon J.-C. Yvard (1968 b) et J.-J. Macaire (1981). Globalement, la taille des sables inférieurs augmente en allant d'est en ouest (figure 2). 


\begin{tabular}{|c|c|c|c|c|c|c|c|}
\hline Formation & $\mathrm{N}^{\circ}$ éch. & Lieu-dit & $\begin{array}{c}\text { Carte IGN au } \\
1 / 50000\end{array}$ & $\begin{array}{c}\text { Coordonnées } \\
\text { Lambert } \\
\text { zone II }\end{array}$ & Altitude (m) & $\begin{array}{l}\text { Profondeur } \\
\text { (m) }\end{array}$ & Lithologie \\
\hline Fx Loir & 32 & Pezou & Selommes & & 90 & 2 & alluvions \\
\hline $\mathrm{Fz}$ & 31 & Monteaux & Montrichard & $506,5-274,8$ & 64 & 1 & alluvions \\
\hline Fx & 33 & Saint-Claude & Blois & $531,7-290,7$ & 85 & 1 & alluvions \\
\hline $\mathrm{Fw}$ & 23 & Choussy & Blois & $518,4-281,4$ & 87 & 2 & alluvions \\
\hline $\mathrm{Fv}_{\mathrm{V}}$ & 29 & Cangey & Amboise & $503-275,2$ & 105 & 1 & alluvions \\
\hline \multirow{4}{*}{$\begin{array}{l}\text { Sables } \\
\text { soufflés }\end{array}$} & 2 & La Gibaudière & Tours & $451,8-277$ & 100 & 0,8 & sables argileux \\
\hline & 3 & Cléré-les-Pins & Tours & $453-272$ & 100 & 0,05 & sables argileux \\
\hline & 6 & $\begin{array}{l}\text { La Renais- } \\
\text { sance }\end{array}$ & Tours & $453,6-266$ & 100 & 0,4 & sables gris \\
\hline & 7 & SW Mazières & Tours & $455,2-265,6$ & 95 & 0,6 & sables argileux \\
\hline \multirow{3}{*}{$\begin{array}{l}\text { Très haute } \\
\text { terrasse Fu }\end{array}$} & 27 & Saint-Ouen & Amboise & $499-274,7$ & 112 & 1 & sables \\
\hline & 9 & N Nazelles & Amboise & $495,4-273,5$ & 105 & 1 & sables argileux \\
\hline & 18 & Dhuizon & Bracieux & $549,1-288,1$ & 129 & 1 & sables argileux \\
\hline \multirow{3}{*}{$\begin{array}{l}\text { Sables de } \\
\text { Mazières }\end{array}$} & 1 & Courcelles & Noyant & $448,5-277$ & 100 & 1 & sables argileux \\
\hline & 4 & W Mazières & Tours & $454,5-266$ & 100 & 1 & sables argileux \\
\hline & 5 & $\begin{array}{l}\text { La Renais- } \\
\text { sance }\end{array}$ & Tours & $453,6-266$ & 100 & 1 & sables argileux \\
\hline \multirow{2}{*}{$\begin{array}{l}\text { Sables de } \\
\text { Château- } \\
\text { Renault }\end{array}$} & 15 & $\begin{array}{c}\text { E Château- } \\
\text { Renault }\end{array}$ & Château-Renault & $495-288,6$ & 115 & 2,5 & sables fins \\
\hline & 17 & $\begin{array}{l}\text { E Château- } \\
\text { Renault }\end{array}$ & Château-Renault & $495-288,6$ & 115 & 1 & $\begin{array}{l}\text { sables gros- } \\
\text { siers }\end{array}$ \\
\hline \multirow{6}{*}{$\begin{array}{l}\text { Sables de } \\
\text { Montreuil }\end{array}$} & 8 & La Chevallerie & Amboise & $494-275$ & 107 & 2 & sables argileux \\
\hline & 26 & La Touche & Blois & $509,2-281,1$ & 108 & 4,5 & sables argileux \\
\hline & 28 & Pierre Bise & Amboise & $497,9-278,5$ & 99 & 5 & sables argileux \\
\hline & 11 & N Neuville & Château-Renault & $492,6-292,5$ & 125 & 0,8 & sables \\
\hline & 12 & Villeporcher & Château-Renault & $499,4-294$ & 122 & 0,8 & sables argileux \\
\hline & 13 & La Bigotière & Château-Renault & $501,4-294,7$ & 128 & 0,8 & sables argileux \\
\hline \multirow{5}{*}{$\begin{array}{l}\text { Sables de } \\
\text { Sologne }\end{array}$} & 46 & N Choussy & Montrichard & $525-264,5$ & 105 & 0,8 & sables argileux \\
\hline & 50 & La Roulerie & Romorantin & $550,5-263,7$ & 105 & 0,8 & sables fins \\
\hline & 51 & La Roulerie & Romorantin & $550,5-263,7$ & 105 & 1 & argile sableuse \\
\hline & 52 & La Grange & Romorantin & $553-263,4$ & 104 & 1 & sables argileux \\
\hline & 53 & La Grange & Romorantin & $553-263,4$ & 104 & 1,5 & argile sableuse \\
\hline \multirow{3}{*}{ Faluns } & 14 & $\begin{array}{c}\text { E Château- } \\
\text { Renault }\end{array}$ & Château-Renault & $495-288,6$ & 115 & 5 & faluns \\
\hline & 42 & E Contres & Romorantin & $537-268,8$ & 117 & 1 & faluns \\
\hline & 43 & E Thenay & Montrichard & $522,6-266$ & 108 & 3 & faluns \\
\hline
\end{tabular}

Tableau 1 : Localisation et lithologie des prélèvements effectués

Fx Loir : alluvions de la basse terrasse de Pezou; Fz : alluvions de la plaine alluviale de la Loire; Fx : alluvions de la basse terrasse Loire; $\mathrm{F}_{w}$ : alluvions de la moyenne terrasse Loire; $\mathrm{Fv}_{\mathrm{v}}$ : alluvions de la haute terrasse Loire.

Location and lithology of samples

Fx Loir: Pezou low terrace alluvial deposits; Fz: Loire alluvial plain; Fx: Loire low terrace; Fw: Loire middle terrace; Fv: Loire high terrace alluvial deposits. 


\section{RÉSULTATS DES ANALYSES SÉDIMENTOLOGIQUES}

\section{Granulométries et conditions de dépôts}

Réalisées avec le granulomètre Cilas HR 850 qui ne traite que la fraction inférieure à $600 \mu \mathrm{m}$, ces mesures permettent de comparer les matériaux fins des dépôts et de calculer les indices de classement $\left(\right.$ So $=\sqrt{ } Q 3 / Q 1$, avec $Q_{3}: 3^{\text {e }}$ quartile et $Q_{1}$ : $1^{\text {er }}$ quartile $)$ et d'asymétrie $\left(\right.$ As $=Q_{1}{ }^{*} Q_{3} /$ Médiane $\left.^{2}\right)$ de P.D. Trask (1932), mais elles ne tiennent pas compte des sables grossiers présents dans la plupart des prélèvements, ce qui peut gêner l'interprétation des modes de mise en place. Huit échantillons représentatifs des différentes formations ont été traités : quatre prélevés dans les « Sables et graviers continentaux » $\left(\mathrm{n}^{\circ} 5,8,15\right.$ et 17 , tableau 1$)$, deux dans les sables soufflés ( $n^{\circ} 3$ et 6 ), un dans les Sables de Sologne ( $\left.\mathrm{n}^{\circ} 46\right)$ et le dernier dans les alluvions de la très haute terrasse de la Loire (n $\left.{ }^{\circ} 9\right)$. Les courbes cumulatives obtenues (figure 3), peuvent être réparties en 3 groupes de sédiments :

- les sables correspondant aux $n^{0}$ 5, 8, 15 et 17 sont mal classés (indice de classement P.-D. Trask supérieur à 3) et renferment plus de $5 \%$ d'argile, mais probablement davantage car la granulométrie à diffraction laser tendrait à minimiser la fraction fine (Levant et al., 1987). Ils présentent deux modes : $450 \mu \mathrm{m}$ et $35 \mu \mathrm{m}$ et la fraction $60-90 \mu \mathrm{m}$ est absente. L'asymétrie négative souligne la prépondérance des particules grossières par rapport à la moyenne. Cette répartition pourrait s'expliquer par une sédimentation en milieu littoral ou un remaniement de tels dépôts : les sables fins qui ont une faible cohésion, selon les courbes de F. Hjulström (1935), auraient été évacués par les courants littoraux alors que les limons de décantation auraient mieux résisté à l'arrachement. Le mauvais classement suggère des épandages effectués par des courants turbulents et spasmodiques. Ils s'apparentent

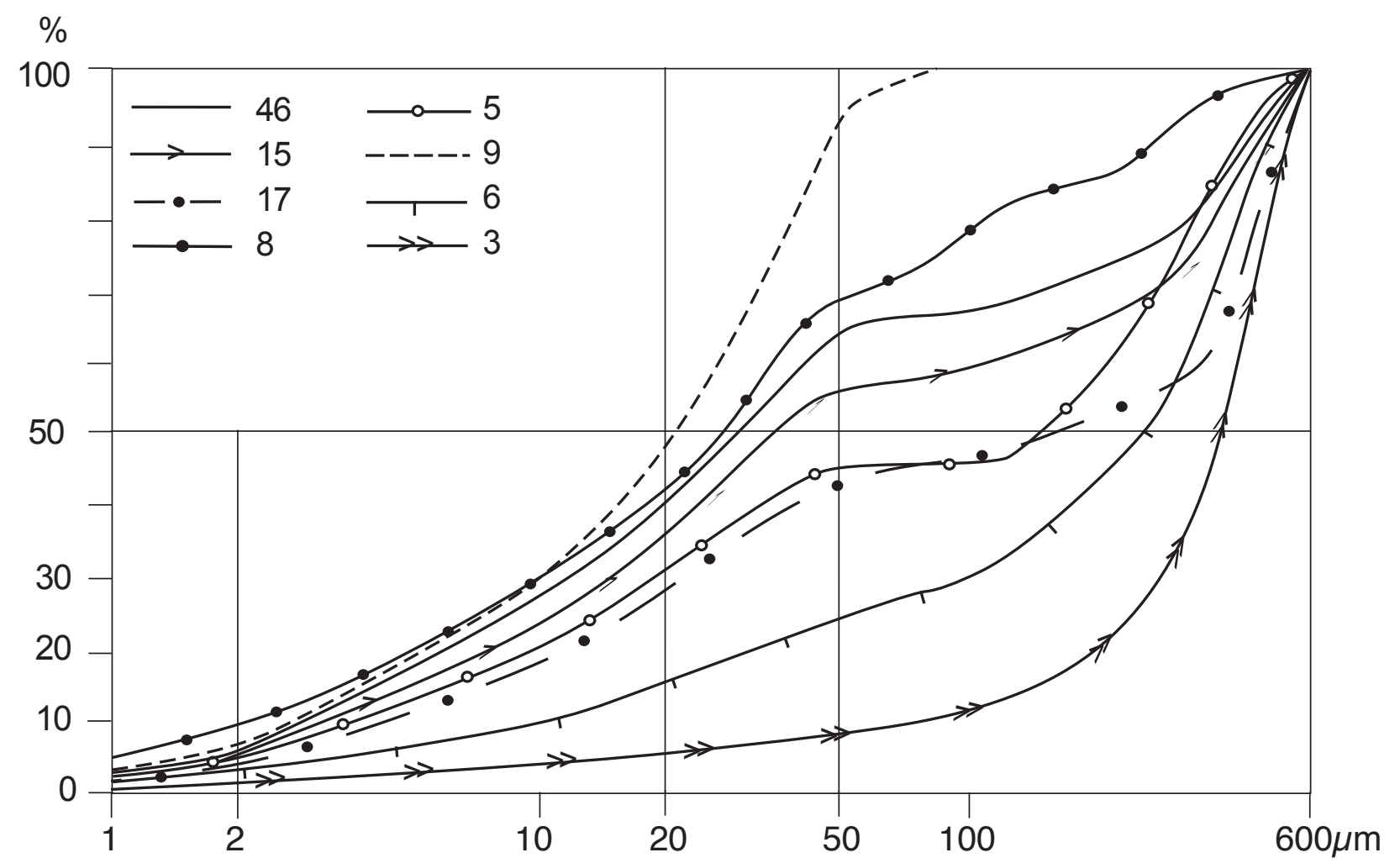

Figure 3 : Courbes granulométriques d'échantillons localisés sur la figure 2

46: Sables de Sologne; 15, 17 : Sables de Château-Renault; 8 : Sables de Montreuil ; 5 : Sables de Mazières; 9 : alluvions Fu de la Loire; 3,6 : sables soufflés.

Grain-size distribution of samples located on fig. 2

46: Sologne sands; 15, 17: Château-Renault sands; 8: Montreuil sands; 5: Mazières sands; 9: Fu Loire alluvia; 3, 6: wind-brought sands. 
aux sables de la bordure occidentale de Sologne $\left(n^{\circ} 46\right)$. En outre, les Sables de Mazières ( $\left.n^{\circ} 5\right)$ et de Château-Renault ( ${ }^{\circ} 15$ et 17) apparaissent plus grossiers que ceux de Montreuil ( $\left.{ }^{\circ} 8\right)$;

- les sables soufflés ( $n^{\circ} 3$ et 6 ) sont nettement mieux classés (indice de classement P.-D.Trask $=1,38$ ) et plus pauvres en argile. Ils présentent des caractéristiques compatibles avec une origine éolienne (Mason et Folk, 1958);

- l'échantillon $n^{\circ} 9$, riche en fines, également bien classé (indice de classement P.-D.Trask = 1,90), avec une asymétrie positive (plus de particules fines que de grossières), pourrait résulter plutôt d'un tri fluviatile (Visher, 1969).

\section{La morphoscopie}

En observant à la loupe binoculaire une centaine de grains, selon la méthode A. Cailleux et J. Tricart (1959), les grains de sables de la fraction 315$500 \mu \mathrm{m}$, obtenue par tamisage, peuvent être classés selon leur morphoscopie en trois catégories :

- les sables soufflés ( $n^{\circ} 3$ et 6 ) montrent une prédominance des grains émoussés-mats $(\mathrm{EM})$ et ronds-mats (RM) (environ $70 \%$ ), fortement éolisés. En effet, les aspects mats prononcés des grains de quartz confirment le phénomène d'éolisation (Yvard, 1968b);

- les Sables de Montreuil ( $\left.{ }^{\circ} 8\right)$, de ChâteauRenault ( ${ }^{\circ} 15$ et 17 ) et du nord de Nazelles ( $n^{\circ} 9$ ) sont constitués de grains à majorité non-usés (NU), plus ou moins colorés par les oxydes de fer. Toutefois, l'échantillon $n^{\circ} 9$ contient également de nombreux émoussés-luisants (environ $35 \%$ ) et l'échantillon $n^{\circ} 8$ renferme quelques grains de quartz issus de ponces limpides caractéristiques des éruptions pyroclastiques rhyolitiques du MontDore (Pastre, 1987 ; Féraud et al., 1990; Pastre et Cantagrel, 2001 ; Pastre, 2004);

- les Sables de Mazières ( ${ }^{\circ} 1,4$ et 5 ) comprennent environ $60 \%$ de grains émoussés-mats (EM) qui traduisent un façonnement marin. Ils sont probablement issus du remaniement de sables marins sénoniens et/ou des Faluns de Touraine helvétiens (Rasplus et al., 1974).

\section{La diffractométrie des rayons $\mathrm{X}$}

Pour cinq échantillons représentatifs des Sables de Château-Renault ( ${ }^{\circ} 15$ et 17), des Sables de Montreuil ( $\left.n^{\circ} 8\right)$, des Sables de Sologne ( $\left.n^{\circ} 53\right)$ et des alluvions de la très haute terrasse de la Loire $\left(n^{\circ} 9\right)$, les poudres de la fraction $<40 \mu \mathrm{m}$ ont été soumises à la diffractométrie des rayons $\mathrm{X}$ afin de déterminer les espèces minérales. Les spectres ont été réalisés avec un diffractomètre de poudre muni d'une sonde à compteurs à scintillation, pour la raie $\mathrm{K}$ alpha du cuivre (figure 4). L'amplitude des pics permet d'évaluer l'importance des principaux minéraux argileux, même si à l'état naturel, la superposition des pics de diffraction de $1^{\mathrm{er}}, 2^{\mathrm{e}}$ et $3^{\mathrm{e}}$ ordres ne facilite pas la distinction des variétés minérales. Les dépôts superficiels entre Loire et Loir présentent une moins bonne cristallinité que les Sables de Sologne ( $\left.\mathrm{n}^{0} 53\right)$. Les smectites dominent plus largement dans les Sables de Château-Renault ( ${ }^{\circ} 15$ et 17) que dans ceux de Montreuil $\left(n^{\circ} 8\right)$ et du nord de Nazelles ( $\left.n^{\circ} 9\right)$. La kaolinite augmente de la profondeur à la surface le long du même profil vertical (nº 15 et 17) sur le site de Château-Renault (figure 2), à cause probablement de l'efficacité de l'altération pédologique postérieure au dépôt de cette formation.

\section{La détermination des minéraux lourds}

Elle a pour objectif de révéler l'origine des principaux flux dans l'espace mais aussi dans le temps. Les fractions comprises entre 75 et $350 \mu \mathrm{m}$, obtenues par tamisage, ont été utilisées pour l'extraction des minéraux lourds. Concentrés selon la méthode classique de séparation au bromoforme (Parfenoff et al., 1970), les minéraux lourds, de densité $>2,89$, ont été déterminés par R. Étienne au microscope optique. Les pourcentages de minéraux transparents ont été calculés à partir de l'analyse d'environ 300 grains. La moyenne de composition établie pour chaque formation révèle des différences sensibles (tableau 2) :

- les Faluns (n 14, 42 et 43) sont caractérisés par l'association zircon-grenat-andalousite-staurotide et tourmaline. Ils se différencient des Sables de Sologne ( ${ }^{\circ} 46,50,51,52$ et 53) qui sont plus riches en zircon et tourmaline et pauvres en grenat. Le zircon et la tourmaline représentent respective- 


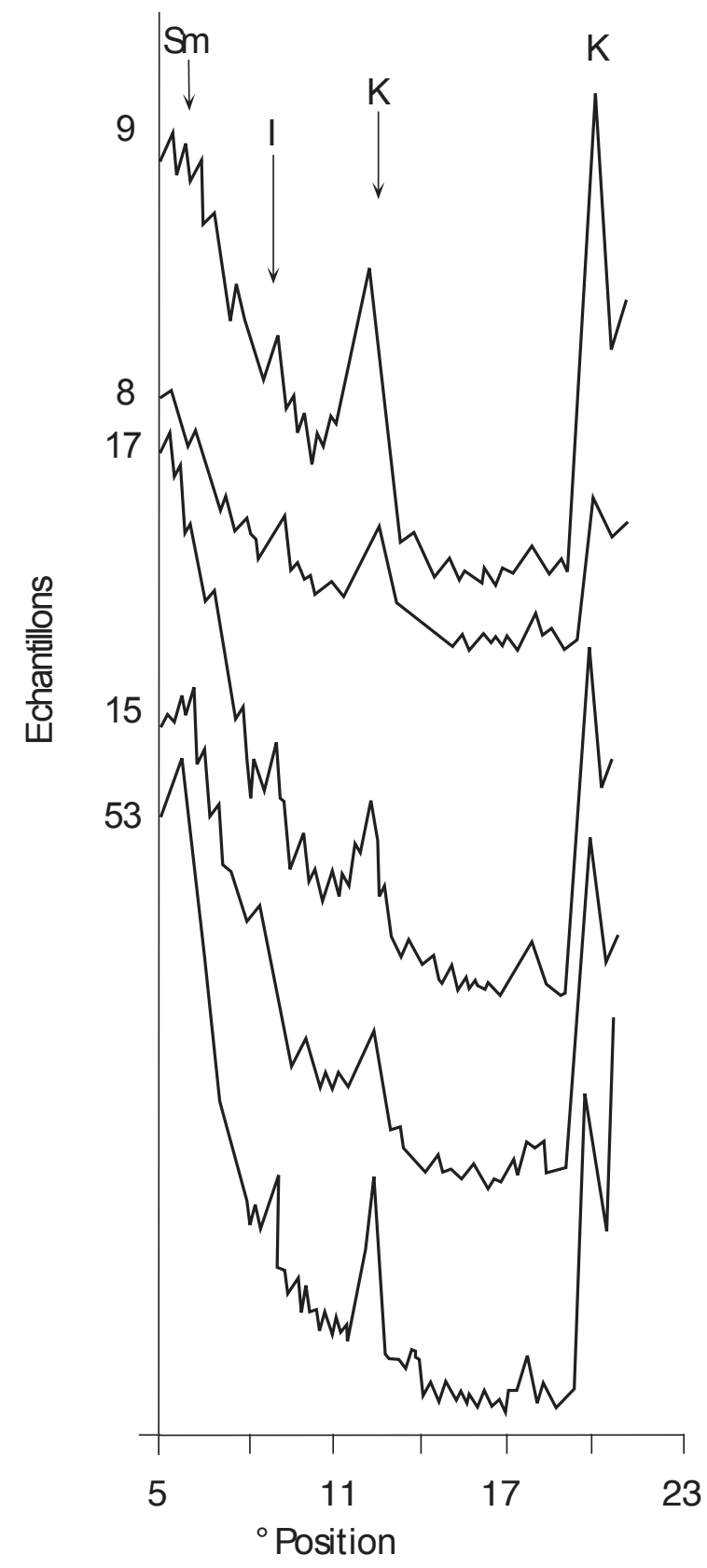

Figure 4 : Spectres de diffraction des rayons X, obtenus sur la fraction $<40 \mu \mathrm{m}$ des échantillons localisés sur la figure 2

I : illite; K : kaolinite; Sm : smectite. 9 : très haute terrasse (Fu) de la Loire; 8 : Sables de Montreuil ; 15, 17 : Sables de Château-Renault; 53 : Sables de Sologne.

$X$ ray diffraction spectra on samples located on fig. 2 I: illite; K: kaolinite; Sm: smectite. 9: very high Loire terrace (Fu); 8: Montreuil sands; 15, 17: Château-Renault sands; 53: Sologne sands.

ment $32 \%$ et $20 \%$ des minéraux lourds dans les Sables de Sologne contre $27 \%$ et $13 \%$ dans les Faluns; le grenat 1,5\% dans les Sables de Sologne contre $12 \%$ dans les Faluns;
- les Sables de Montreuil ( $n^{\circ} 8$ et 28) et de Château-Renault (no 15 et 17) contiennent de la staurotide, du zircon, de la tourmaline et de l'andalousite, mais sont très pauvres en grenat. Sur un même profil vertical, on peut relever, de la profondeur à la surface, l'augmentation de la staurotide et de la tourmaline et la diminution du zircon. De plus, l'échantillon $n^{\circ} 26$ renferme un faible pourcentage de minéraux volcaniques : clinopyroxène et amphibole brune;

- les Sables de Mazières ( $\left.{ }^{\circ} 4,5\right)$ sont dominés par la staurotide et la tourmaline (respectivement $37 \%$ et $39 \%$ ). Ils n'ont ni grenat, ni zircon. Ils renferment un pourcentage de minéraux volcaniques légèrement plus fort que les Sables de Montreuil;

- les dépôts de la très haute terrasse au nord de Nazelles $\left(n^{\circ} 9,27\right)$ sont en revanche très riches en zircon (plus de $60 \%$ ) et renferment quelques grains de clinopyroxènes, ce qui indique des apports du Massif central ;

- les sables soufflés (n³,6) renferment des pourcentages notables de minéraux volcaniques, en particulier des pyroxènes et quelques amphiboles brunes.

Dans la vallée de la Loire, les alluvions sont caractérisées par la présence de minéraux volcaniques dont les pourcentages augmentent en général des nappes les plus anciennes aux plus récentes. Les alluvions de la terrasse $\mathrm{F}_{V}$ ( $\left.\mathrm{n}^{\circ} 29\right)$ renferment moins de zircon que celles de la très haute terrasse $\left(n^{\circ} 9\right)$ et un faible pourcentage d'amphiboles brunes et de clinopyroxènes. En revanche les alluvions des terrasses $\mathrm{F}_{\mathrm{W}}$ et $\mathrm{Fx}$ sont caractérisées par une forte teneur en minéraux lourds volcaniques : les amphiboles brunes l'emportent sur les clinopyroxènes dans la moyenne terrasse ( $\left.n^{\circ} 23\right)$, alors que l'inverse se produit pour la basse terrasse (no 33). L'olivine apparaît dans les alluvions Fx et donne $30 \%$ des minéraux lourds de la plaine alluviale $\mathrm{Fz}\left(\mathrm{n}^{\circ} 31\right)$.

\section{Identification de plusieurs nappes détritiques}

La confrontation des résultats obtenus par les méthodes précédentes peut déboucher sur l'identification de plusieurs nappes détritiques :

- Les Sables de Château-Renault ( $\left.n^{\circ} 15,16,17\right)$ et de Montreuil ( $\left.\mathrm{n}^{\circ} 8,11,12,13,26,28\right)$ se ressemblent par leur mauvais classement, leur forte 


\begin{tabular}{|c|c|c|c|c|c|c|c|c|c|c|c|c|c|c|c|c|c|c|c|c|c|c|}
\hline 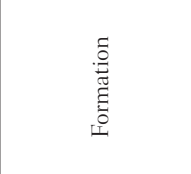 & 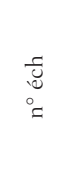 & $\begin{array}{l}\tilde{0} \\
\stackrel{0}{N}\end{array}$ & 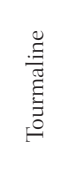 & 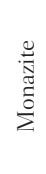 & 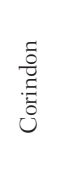 & 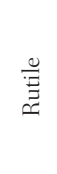 & 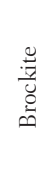 & 节 & 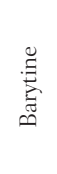 & 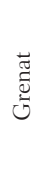 & 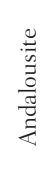 & 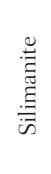 & 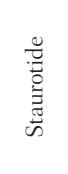 & 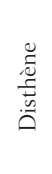 & 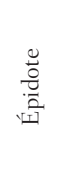 & 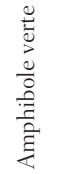 & 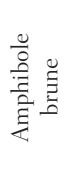 & 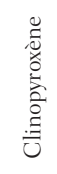 & 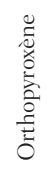 & $\frac{\text { 芯 }}{\frac{0}{\tilde{n}}}$ & $\stackrel{\mathscr{\Xi}}{\stackrel{\Xi}{二}}$ & 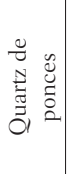 \\
\hline Fx Loir & 32 & 13 & 27 & & & 2 & & & & 2 & 5 & & 46 & 5 & & & & & & & & \\
\hline $\mathrm{Fz}$ & 31 & 7 & 2 & & & 0,5 & & & & 8 & & & & 3 & & 1 & 1,5 & 44 & 4 & 1 & 28 & \\
\hline Fx & 33 & 1 & 1 & & & & & & & 3 & 1 & 0,5 & 1 & & & 1 & 5 & 67,5 & 7 & 1 & 11 & \\
\hline$F_{W}$ & 23 & 22 & 9 & & 0,5 & & & & & 7 & 2,5 & 4 & 10 & 3 & & 8 & 14 & 8 & 6 & 5,5 & & \\
\hline $\mathrm{Fv}_{\mathrm{V}}$ & 29 & 45 & 11 & 1,5 & 1 & 3,5 & & & & 1 & 14 & 1,5 & 15 & 1 & & 1,5 & 1 & 2 & & 0,5 & & \\
\hline \multirow{5}{*}{ Sables soufflés } & 2 & 11 & 25 & & & 0,5 & & & & 5,5 & 13 & & 39 & & & 1,5 & & 1,5 & 2 & 0,5 & & \\
\hline & 3 & 7 & 24 & & & 1,5 & & & & 7 & 5 & 1 & 36 & & & 1 & 1,5 & 12 & 2,5 & 1,5 & & \\
\hline & 6 & 4 & 29 & & & 1,5 & & & & 8 & 10 & & 40 & & & & 1 & 4 & 2 & & & \\
\hline & 7 & 4 & 23 & & & & & & & 7 & 13 & & 40 & & & & & 10 & 2,5 & & & \\
\hline & moy & 6 & 25 & & & 1 & & & & 7 & 9,7 & 0,2 & 38 & 2,5 & & 0,6 & 0,5 & 7 & 2 & 0,5 & & \\
\hline \multirow{4}{*}{$\begin{array}{l}\text { Très haute } \\
\text { terrasse Fu }\end{array}$} & 27 & 55 & 4 & & 0,5 & 3,5 & & & & 1 & 3,5 & 2 & 28 & 2 & & & & 0,5 & & & & \\
\hline & 9 & 68 & 15 & & & 2 & & & & & 2 & 3,5 & 7 & 1,5 & & & & 0,5 & & & & \\
\hline & 18 & 40 & 29 & & & 3 & & 1 & & 2 & 11 & 4 & 6 & 1 & & & & 2 & & & & \\
\hline & moy & 54 & 15 & & 0,2 & 3 & & 0,3 & & 1 & 5 & 3,2 & 16,5 & 1,5 & & & & 1 & & & & \\
\hline \multirow{3}{*}{$\begin{array}{l}\text { Sables de } \\
\text { Mazières }\end{array}$} & 4 & & 24 & & & 2 & & & & & 11 & 1 & 50 & 2 & & & 1 & 8 & 2 & & & \\
\hline & 5 & & 55 & & & 2 & & & & & 15 & & 25 & 3 & & & & & & & & \\
\hline & moy & & 39 & & & 2 & & & & & 13 & 1 & 37 & 2,5 & & & 0,5 & 4 & 1 & & & \\
\hline \multirow{3}{*}{$\begin{array}{l}\text { Sables de } \\
\text { Château-Renault }\end{array}$} & 15 & 44 & 14 & 2 & & 5 & & & & 3 & 7 & 0,5 & 22 & 2 & 0,5 & & & & & & & \\
\hline & 17 & 14 & 23 & & & 2,4 & 0,8 & & & & 17 & 0,8 & 40 & 2 & & & & & & & & \\
\hline & moy & 29 & 18 & 1 & & 4 & 0,4 & & & 2 & 12 & 0,6 & 31 & 2 & 0,2 & & & & & & & \\
\hline \multirow{7}{*}{$\begin{array}{l}\text { Sables de } \\
\text { Montreuil }\end{array}$} & 8 & 29 & 15 & & & 6 & & & & & 9 & 6 & 34 & 1 & & & & & & & & $*$ \\
\hline & 26 & 33 & 23 & 1 & 0,5 & 5 & 1 & & & 0,5 & 11 & & 21 & 3 & & 0,5 & 0,5 & 0,5 & & & & $*$ \\
\hline & 28 & 38 & 16 & & 0,5 & 3,6 & & & & & 9,5 & 0,5 & 26 & 6 & & & & & & & & \\
\hline & 11 & 35 & 18 & & & 4 & 3,5 & & & & 12 & & 25 & 2,5 & & & & & & & & \\
\hline & 12 & 20 & 22 & 0,5 & & 2,5 & 1,5 & & & 2,5 & 14 & 0,5 & 33 & 2,5 & 1,5 & & & & & & & \\
\hline & 13 & 45 & 18 & 1 & 0,5 & 4 & 0,5 & & & & 6 & 4 & 20 & 1 & & & & & & & & $*$ \\
\hline & moy & 33 & 18,5 & 0,5 & 0,3 & 4 & 1 & & & 1 & 10 & 2,5 & 27 & 2,5 & 0,3 & & & & & & & \\
\hline \multirow{6}{*}{ Sables de Sologne } & 46 & 32 & 22 & 0,6 & 0,5 & 4,5 & 1,3 & & & 3,4 & 7 & 3,4 & 22 & 2,7 & 0,6 & & & & & & & \\
\hline & 50 & 15 & 25 & 0,7 & 0,7 & 2,5 & 0,7 & & & 1,5 & 18 & 1,5 & 32 & 1,5 & 0,7 & & & & & & & \\
\hline & 51 & 21 & 26 & & 1 & 2,5 & & & & 1 & 14 & 2,3 & 26 & 3,9 & 0,7 & & & & & 1,5 & & \\
\hline & 52 & 42 & 15 & 0,4 & 0,8 & 3,2 & 0,8 & 0,8 & 0,7 & 1,6 & 4,8 & 2 & 24 & 1,6 & 0,4 & & 0,4 & & & 1,6 & & \\
\hline & 53 & 50 & 14 & 1 & & 3,3 & 1 & & 2,3 & 1 & 4,5 & 2,3 & 19 & 1,5 & & & & & & & & \\
\hline & moy & 32 & 20 & 0,6 & 1 & 3 & 0,6 & 0,1 & 0,6 & 2 & 9,7 & 2,3 & 25 & 2,2 & 0,5 & & 0 & & & 0,6 & & \\
\hline \multirow{4}{*}{ Faluns } & 14 & 39 & 9 & 1 & & 5 & & & & 15 & 15 & 1 & 14 & 1 & & & & & & & & \\
\hline & 42 & 18 & 12 & & 0,5 & 4 & 0,5 & 0,5 & & 8 & 16 & 5,4 & 30 & 3 & 2,1 & & & & & & & \\
\hline & 43 & 24 & 20 & & 0,5 & 2,5 & & & & 13 & 12 & 2 & 23 & 2,5 & 0,5 & & & & & & & \\
\hline & moy & 27 & 13,5 & 0,3 & 0,5 & 3,5 & 0,3 & 0,3 & & 12 & 14 & 2,8 & 22 & 2 & 1,3 & & & & & & & \\
\hline
\end{tabular}

Tableau 2 : Composition en minéraux lourds des échantillons localisés sur la figure 2 et le tableau 1

Fx Loir : alluvions de la basse terrasse de Pezou; Fz : alluvions de la plaine alluviale de la Loire; Fx : alluvions de la basse terrasse Loire; FW : alluvions de la moyenne terrasse Loire; Fv : alluvions de la haute terrasse Loire.

Heavy minerals distribution on samples located on fig. 2 and table 1

Fx Loir: Pezou low terrace allwvial deposits; Fz: Loire allwvial plain; Fx: Loire low terrace; Fw: Loire middle terrace; Fv: Loire high terrace alluvial deposits. 
altération et leur composition en minéraux lourds : richesse en staurotide et absence du grenat. La présence de quartz de ponces dans les échantillons $n^{\circ} 8, n^{\circ} 26$ et probablement $n^{\circ} 13$ daterait cette formation du Pliocène supérieur (Tourenq et Turland, 1982, Tourenq, 1989) et plus précisément de 3,1 Ma (Féraud et al., 1990; Nomade et al., 2014a et b). Soumis à la spectrométrie Mössbauer, l'échantillon $\mathrm{n}^{\circ} 8$ contient $73 \%$ d'hydroxydes de fer parmi le fer total, chiffre voisin de ceux obtenus pour les épandages placés dans le Pliocène supérieur entre Angers et Laval (Larue et Grenèche, 1994).

- Les Sables de Mazières ( $\mathrm{n}^{\circ} 1,4$ et 5 ) sont issus du remaniement de dépôts marins locaux ainsi que l'attestent la morphoscopie des grains et l'absence de grenat et de zircon (tableau 2), minéraux provenant surtout du Massif central. Les minéraux dominants (staurotide et tourmaline) abondent dans le substrat sénonien (Rasplus et al., 1974).

- Les alluvions du nord de Nazelles, mieux classées, très riches en zircon témoignent d'un transport fluviatile lointain, leur forte altération souligne une longue exposition à des conditions lessivantes favorisées par l'encaissement fluviatile.

- Les sables soufflés ( $n^{\circ} 3$ et 6) sont caractérisés par l'abondance des grains RM et des minéraux lourds d'origine volcanique apportés par le vent à partir des alluvions du Val de Loire (tableau 2).

\section{ENSEIGNEMENTS PALÉOGÉOGRAPHIQUES}

\section{Des apports en provenance de sources multiples}

Liés à la lithologie des bassins versants, les apports des cours d'eau peuvent être caractérisés par leurs cortèges de minéraux lourds. Cependant, ces derniers présentent des différences notables en fonction de l'âge des dépôts, suite à la progression de l'altération avec le temps, mais aussi en raison de possibles modifications de l'aire des bassins-versants. Selon J.-J. Macaire (1983), les alluvions de la Loire sont caractérisées par la présence de pyroxènes et d'olivine, celles du Cher par l'association amphibole-épidote-grenat et par un rapport andalousite/ staurotide inférieur à 1 , celles de l'Indre par la présence de grenat et par un rapport andalousite/ staurotide supérieur à 1 . Les cours d'eau descen- dant du Perche sont riches en staurotide, tourmaline et andalousite ainsi que le montrent les apports du Loir (éch. no 32). En se limitant aux formations alluviales les plus anciennes, du Pliocène supérieur et du Pléistocène inférieur et moyen, les apports du Cher sont caractérisés par la prédominance du zircon et par la présence de monazite et de brookite issues des roches cristallines du Massif central. Les minéraux d'origine métamorphique (andalousite principalement) marquent les flux de l'Indre, le zircon, la staurotide et l'andalousite caractérisent ceux de la Loire.

Le tableau n ${ }^{\circ} 2$ montre que les «Sables et graviers continentaux » ont un rapport andalousite/ staurotide inférieur à 1 , ce qui traduit des apports importants par l'axe du Cher. Néanmoins, on trouve aussi des minéraux traceurs caractéristiques des autres axes fluviaux. Ceci suggère que l'alimentation des «Sables et graviers continentaux » n'a pas été effectuée par un seul paléo-cours, mais que tous les organismes drainant la gouttière centrée sur la Loire y ont participé dans des proportions variables (figure 5).

\section{Du Miocène supérieur au Pliocène supérieur : mise en place des «Sables et graviers continentaux »}

Les Sables de Château-Renault, qui ravinent les Faluns et remplissent en partie la ría préfalunienne de la Brenne (figure 2), pourraient traduire des apports locaux du fait de leur mauvais classement. L'absence de grenat, minéral présent dans les Faluns et dans les Sables de Sologne, peut s'expliquer pour une partie par la forte altération météorique qu'ils ont subie. De la profondeur à la surface le long d'un même profil vertical, la diminution du pourcentage de zircon et l'augmentation de ceux de la staurotide et de la tourmaline indiquent que les dépôts proviennent de l'érosion de substrats de moins en moins éloignés : en effet le zircon provient surtout du Massif central et des formations qui en sont issues, alors que la staurotide et la tourmaline sont abondantes dans les terrains avoisinants, comme les craies et sables du Sénonien.

Plus récents que les Sables de Sologne qui sont situés pour une partie sous les Faluns (Ginsburg et Janvier, 1970, Dewolf, 1982), les Sables de Montreuil ont été qualifiés de post-helvétiens par C. Klein 


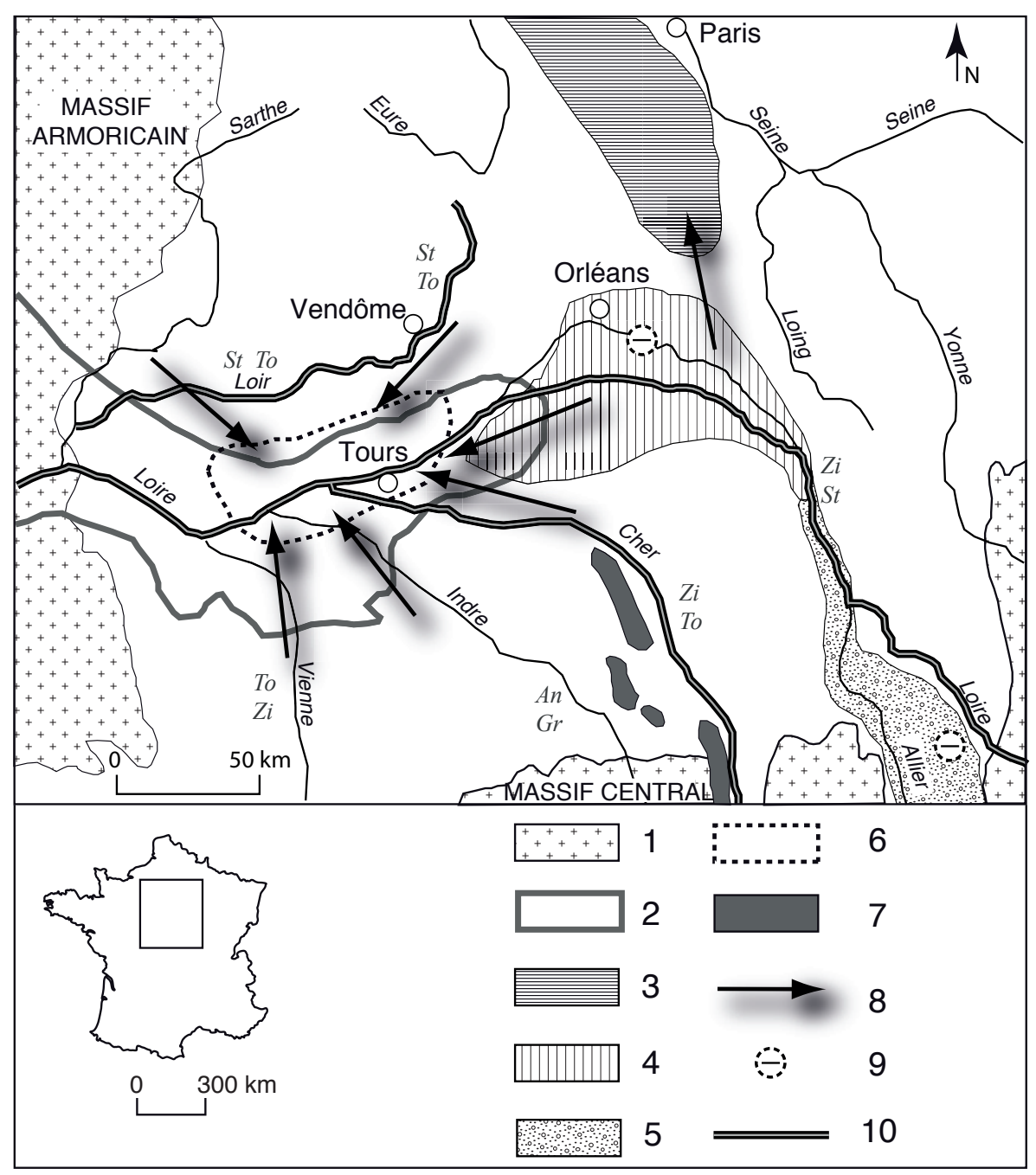

Figure 5 : Principaux paléoflux du Miocène supérieur au Pléistocène inférieur

1 : massif ancien; 2 : extension de la Mer des Faluns de Touraine; 3 : Sables de Lozère (Burdigalien inférieur à Pliocène supérieur); 4 : Sables et argiles de Sologne (Miocène moyen à Pliocène supérieur); 5 : Sables et argiles du Bourbonnais (Pliocène supérieur); 6 : Sables et graviers continentaux de Touraine (Miocène supérieur à Pliocène supérieur); 7 : témoins alluviaux de l'axe du Cher (Pliocène supérieur); 8 : épandages (Miocène supérieur à Pliocène supérieur) et minéraux lourds prédominants (An : andalousite, Gr : grenat, $\mathrm{St}$ : staurotide, To : tourmaline, $\mathrm{Zi}$ : zircon); 9 : cuvette subsidente; 10 : cours chenalisé et nappe alluviale de $1,1 \mathrm{Ma}$. Main palaeoflows from $\mathrm{Up}_{\mathrm{p}}$ per Miocene to Lower Pleistocene 1: basement; 2: Touraine Mer des Faluns; 3: Lozère sands (Lower Burdigalian to Upper Pliocene); 4: Sologne sands and clays (Middle Miocene to Upper Pliocene); 5: Bourbonnais sands and clays (Upper Pliocene); 6: Touraine sands and gravels (Upper Miocene to Upper Pliocene); 7: Cher detritic deposits (Upper Pliocene); 8: detritic flows (Upper Miocene to Upper Pliocene) and main heavy minerals (An:andalusite, Gr: garnet, St : staurolite, To: tourmaline, $\mathrm{Zi}$ : zircon); 9: subsidence; 10: chenalised flow and allwial deposits of $1.1 \mathrm{My}$.
(1973) et par C. Mégnien et al. (1980). Ils ressemblent aux Sables de Sologne par leur richesse en feldspaths et leur charge en graviers et galets d'origine locale (Rasplus, 1978). La découverte par G. Lecointre (1931) de lentilles de marne compacte " présentant des empreintes de coquilles des Faluns » sous les Sables de Montreuil au nord-ouest de Nazelles tend à prouver qu'ils sont issus pour une part de la dissolution des Faluns. Cette thèse est reprise par A. Vatan (1947) qui insiste sur l'efficacité des karstifications post-helvétiennes, mais qui remarque aussi l'importance des faciès de transport dans les Sables de Montreuil. G. Denizot (1927) signale la présence de chailles nivernaises aux côtés de silex régionaux et suggère un raccordement aux « cailloutis préligériens » qui traversent la Sologne. Les cailloutis situés au nord du Beuvron renferment quelques minéraux volcaniques (échantillon $n^{\circ} 18$ ) qui témoigneraient d'apports par la Loire amont. Ils pourraient être le prolongement des « Sables et argiles du Bourbonnais » selon D. Cruz Mermy et al. (2007). La découverte de grains de quartz issus de ponces, certains dans les échantillons $\mathrm{n}^{\circ} 8$ et 26, rajeunit fortement ces dépôts ou une partie d'entre eux, mais ne permet pas de mettre en évidence un écoulement direct de la Loire vers l'Atlantique au Pliocène supérieur. En effet, l'absence de grenat et des autres minéraux caractéristiques des nappes pliocènes en provenance du Massif central, comme l'amphibole brune et le sphène, semble interdire un transport lointain par la Loire-amont. Toutefois, l'altération peut avoir entraîné la disparition de ces minéraux (Pastre, 1986). Le mauvais classement des sédiments est un argument supplémentaire. Les dimensions des matériaux, qui augmentent localement vers l'ouest pour les Sables de 
Mazières peuvent suggérer des transports s'effectuant d'ouest en est et non vers l'Atlantique, cependant il peut s'agir de variations latérales de faciès liées à la distance par rapport au centre du chenal. Les coupes observées ne permettent pas de déterminer avec certitude l'orientation des chenaux. De par leur composition en minéraux lourds, les Sables de Mazières sont probablement plus récents que ceux de Montreuil car ils renferment un plus fort pourcentage de minéraux volcaniques que ces derniers. Toutefois, des datations numériques seraient utiles, comme celles obtenues par la méthode de la résonance de spin électronique (ESR) appliquée aux grains de quartz fluviatiles blanchis optiquement (Despriée et al., 2007; Voinchet et al., 2007 ; Tissoux et al., 2011).

Depuis la régression post-helvétienne jusqu'au Pliocène supérieur, l'érosion semble l'avoir emporté sur l'accumulation. La morphogenèse s'est limitée à des remaniements successifs, incorporant des matériaux issus de substrats de plus en plus locaux. Une telle évolution impose une relative stabilité tectonique pour cette région tourangelle, alors que la Sologne voisine a subi des déformations tectoniques notables (Étienne et Larue, 2011). Toutefois, de petites subsidences locales ou des phénomènes karstiques sont nécessaires pour expliquer le piégeage des dépôts en transit sur la surface acyclique (Dewolf et Pomerol, 1997), en constant réaménagement, du Miocène supérieur au Pliocène supérieur. Les remaniements et accumulations du Pliocène supérieur correspondent à une période de refroidissement et de baisse du niveau marin selon les courbes isotopiques (Lisiecki et Raymo, 2005). Des systèmes d'épandages semblables auraient fonctionné dans le Bassin parisien jusqu'à l'encaissement des cours d'eau (Freytet et al., 1989). Des remaniements se sont aussi produits en Sologne, ils expliqueraient les âges d'environ 700 ka obtenus pour la partie supérieure des Sables et argiles de Sologne (Tissoux et al., 2013).

Le déversement de la Loire vers l'Atlantique, que C. Passerat (1910), G. Denizot (1927), J. Gras (1963) plaçaient lors de la transgression de la Mer des Faluns, a été rajeuni au Miocène supérieur par J.-C. Yvard (1968 a) qui a mis en évidence du Miocène continental post-helvétien dans la Champeigne tourangelle, et par J. Tourenq et al. (1971) qui démontrent avec des arguments minéra- logiques que les Faluns n’ont pas été alimentés par des apports de la Loire-amont, mais plutôt à partir du Massif armoricain. Les caractéristiques sédimentologiques des sables «post-helvétiens » suggèrent que les apports directs par une paléo-Loire chenalisée n'existaient pas au Pliocène supérieur. Il faut attendre le creusement précédant l'accumulation de la nappe alluviale Fu du nord de Nazelles pour que cela se produise (figure 5).

\section{Au Pléistocène : l'encaissement des cours d'eau}

Étudiées par J.-J. Macaire (1983) en Touraine, les terrasses pléistocènes de la Loire sont beaucoup moins étendues qu'en Sologne (Larue et Étienne, 2001 ; Étienne et Larue, 2011). La nappe Fu des alluvions de la Loire ( $n^{\circ} 9$ et 27) ne forme pas une terrasse très régulière en raison de sa dissection ultérieure, néanmoins, elle nous indique que le creusement postérieur à la mise en place des Sables de Montreuil est resté très limité : les alluvions Fu sont emboîtées dans ces derniers. Résultant de mouvements épirogéniques positifs, le creusement des vallées débute, selon J.-J. Macaire (1983 et 1984), à des âges différents selon les secteurs considérés : au Quaternaire ancien en Touraine, mais dès le Pliocène ancien dans le Châtelleraudais. Faute d'affleurements suffisants dans les dépôts $\mathrm{Fu}$, on ne peut affirmer qu'il s'agisse de la première nappe d'origine périglaciaire. Ne contenant que de rares minéraux lourds volcaniques, cette nappe n'est pas le prolongement du fleuve à clinopyroxènes daté de 1,75 Ma qui, selon J. Tourenq et C. Pomerol (1995), rejoignait la Seine à l'aval de Paris. Elle semble se raccorder avec la terrasse Fu qui traverse la Sologne au nord du Beuvron et qui renferme un cortège minéralogique très semblable (éch. $\mathrm{n}^{\circ} 18$ ). Au nord de Tours, elle correspond à la terrasse de 60 m décrite par E. Chaput (1917). Cette nappe correspond à plus haute nappe alluviale datée de $1,1 \pm 0,14 \mathrm{~mA}$ dans la vallée du Loir (Tissoux et al., 2011) et de 1,1 $\pm 0,4 \mathrm{~mA}$ en Sologne (Tissoux et al., 2013). Les courbes isotopiques de L.-E. Lisieki et M.-E. Raymo (2005) indiquent que l'accumulation correspond à une phase de refroidissement et de baisse du niveau marin. De Blois à Nazelles, cette nappe domine la haute terrasse $\mathrm{Fv}_{\mathrm{v}}$ qui se tient à une quarantaine de mètres d'altitude relative par rapport 
au lit mineur de la Loire. Les terrasses inférieures $\left(\mathrm{FW}_{\mathrm{w}}, \mathrm{Fx}\right)$ sont toutes caractérisées par une forte teneur en minéraux lourds volcaniques.

Au Pléistocène récent, probablement durant les phases froides défavorables à la végétation, les vents ont transporté depuis les alluvions weichséliennes $(\mathrm{Fx})$ du Val de Loire, des sables riches en éléments volcaniques qui aujourd'hui recouvrent des substrats variés (Yvard, 1968b). Localement, ces sables éolisés seraient mêlés à des dépôts pliocènes, comme le suggère le moins bon classement de l'échantillon $n^{\circ} 6$.

\section{Conclusion}

L'analyse sédimentologique favorise la distinction de différentes nappes au sein des «Sables et graviers continentaux », mais leur âge reste hypothétique, en l'absence de datations absolues. La méthode de la résonance de spin électronique (ESR) pourrait probablement combler ces lacunes. En effet, seule la présence de grains de quartz issus de ponces, émis par le Mont-Dore au Pliocène supérieur, permet d'affirmer que la partie supérieure remaniée des Sables de Montreuil est contemporaine de la mise en place de l'épandage des Sables du Bourbonnais décrit et daté par J. Tourenq (1989). En revanche, les autres caractéristiques sédimentologiques des dépôts vont plutôt à l'encontre de l'hypothèse d'un écoulement direct de la Loire-amont vers l'Atlantique à cette époque. La localisation de ces dépôts grossiers en Sologne et en Touraine indique aussi que les "Sables et argiles du Bourbonnais », transportés par des écoulements divagants, se sont étalés sur une très vaste surface, du Bourbonnais à la Touraine. Ils ont été préservés dans des cuvettes subsidentes de taille inégale qui étaient reliées entre elles par ces écoulements intermittents (figure 5). Le déversement fluviatile définitif ne se serait donc produit que lors du faible creusement précédant l'accumulation de la nappe alluviale résiduelle conservée au nord de Nazelles.

Issues essentiellement des substrats locaux, ces formations détritiques azoïques, conservées dans des plans très voisins, attestent une relative stabilité tectonique de la région tourangelle, contrairement à la Sologne voisine qui a connu des déformations notables.

\section{Remerciements}

Nous remercions Robert Étienne, professeur honoraire, qui s'est chargé de la détermination des minéraux lourds, Anne-Marie Mercier (URA 449, Laboratoire des fluorures, université du Maine) qui a effectué l'analyse des minéraux argileux et Jean-Jacques Pechon qui a réalisé les granulométries avec le granulomètre HR 850 de l'IUT Chimie du Mans. Cet article a bénéficié des corrections et remarques constructives de S. Molliex, J.-F. Pastre, G. M. Maillet et de deux relecteurs anonymes.

\section{Bibliographie}

Alcaydé G., 1968. Carte géologique de France au 1/50000, feuille Amboise ( $\mathrm{n}^{\circ}$ 458), Notice explicative 13 p., BRGM, Orléans.

André J.-P., Biagi R., Moguedet G., Buffard R., G. Clément G., F. Redois F., Baloge P.-A., 2003. Dépôts carbonatés et siliciclastiques sur une plate-forme marine : les faluns miocènes d'Anjou, Annales de Paléontologie, vol. 89, p. 113-123.

Cailleux A., Tricart J., 1959. Initiation à l'étude des sables et des galets, CDU, (3 fasc. I : 376 p., II : 194 p., III : 202 p.), Paris.

Cavelier C., Clozier L., Debrand-Passard S., Pomerol C., Tourend J., 1993. Les écoulements fluviatiles successifs issus du Massif Central dans le Bassin Parisien, tributaires de l'Atlantique ou de la Manche au Néogène-Pléistocène : relations avec la tectonique, Livre des résumés du colloque "Géologie de la France» (Paris), Édit. Société Géologique de France et BRGM, 2 p.

Chaput E., 1917. Recherches sur les terrasses alluviales de la Loire et de ses principaux affluents, Annales de l'Université de Lyon, nouvelle série, I, fasc. 41, 303 p.

Courville P., Crônier C., 2003. Les faluns tortoniens (Miocène supérieur) de Noyant-La-Plaine (Ouest de la France). Nouvelles données lithologiques et paléontologiques, Annales de la Société Géologique du Nord, vol. 10, p. 275283.

Cruz Mermy D., Giot D., Maget P., Charnet F., GuillouFrottier L., Jaudin F., Irribaria R., Delétang H., MagGIORAni L., 2007. Notice explicative, carte géologique de la France à 1/50000, feuille Bracieux ( $n^{\circ} 429$ ), Orléans, Édit. BRGM, $171 \mathrm{p}$

Denizot G., 1927. Les formations continentales de la région orléanaise, Thèse Sciences, Vendôme, Launay et fils Impr., $582 \mathrm{p}$.

Despriée J., Voinchet P., Bahain J.-J., Tissoux H., Falguères C., Dépont J., Dolo J.-M., 2007. Les nappes alluviales pléistocènes de la vallée moyenne du Cher (Région Centre, France) - Contexte morphosédimentaire, chronologie ESR et Préhistoire - Premiers résultats, Quaternaire, vol. 18, $n^{\circ} 4$, p. 349-368. 
Dewolf Y., 1982. Le contact Île-de-France-Basse Normandie, évolution géodynamique, Mémoires et Documents de Géographie, CNRS, $253 \mathrm{p}$.

Dewolf Y., Pomerol C., 1997. Surfaces d'érosion polygéniques dans les formations cénozoïques du Bassin parisien, Bulletin de la Société Géologique de France, t. 168, n 4, p. 473-482.

Dewolf Y., Penven M.-J., 2007. Les grands épandages détritiques des marges nord du Massif Central, in Du continent au bassin versant, livre en hommage au professeur Alain Godard, Presses Université Blaise Pascal, ClermontFerrand, p. 121-131.

Étienne R., Larue J.-P., 2011 . Contribution à l'étude des liaisons Loire-Seine : mise en évidence par l'étude des minéraux lourds de l'antécédence de la Loire en Sologne (Bassin parisien, France), Physio-Géo, Géographie Physique et Environnement, vol. 5, p. 269-292.

Féraud G., Lo Bello P., Hall C., Cantagrel J.-M., York D., Bernat M., 1990. Direct dating of Plio-Quaternary pumices by $40 \mathrm{Ar} / 39 \mathrm{Ar}$ step-heating and single grain laser fusion methods: the example of the Monts-Dore massif (Massif central, France), Journal of Volcanic and Geothermal Research, vol. 40, p. 39-53.

Freytet P., Dewolf Y., Joly F., Plet A., 1989. L'évolution de la section Loire-Loing- Seine à la fin du Tertiaire. Réinterprétation géomorphologique des relations entre les sables de Sologne et ceux de Lozère. Signification du complexe alluvial de la Montagne de Trin, Bulletin d'Information des Géologues du Bassin de Paris, vol. 26, n 2, p. 49-57.

Ginsburg L., Janvier P., 1970. Présence de sables helvétiens d'origine fluviatile sous les faluns du Bassin de Noyant-sousle Lude, Bulletin du Muséum National d'Histoire Naturelle, $2^{\mathrm{e}}$ série, t. 42, nº 2, p. 435-439.

Gras J., 1963. Le Bassin de Paris méridional, étude géomorphologique, Thèse d'État de Lettres, Rennes, 494 p.

Huulström F., 1935. Studies of the morphological activity of rivers as illustrated by the river Fyris, Bulletin of the Geological Institute, University of Uppsala, n 25, p. 221-527.

KLein C., 1973. Massif Armoricain et Bassin Parisien. Contribution à l'étude géologique et géomorphologique d'un massif ancien et de ses enveloppes sédimentaires, thèse Doct. État, Paris, Louis Jean Imp., Gap, 882 p.

KLEIN C., 1990. L'évolution géomorphologique de l'Europe hercynienne occidentale et centrale : aspects régionaux et essai de synthèse, Mémoires et Documents de Géographie, Ed. CNRS, 177 p., + doc. h. t.

LARue J.-P., GrenÈche J.-M., 1994. Les dépôts détritiques pliocènes du contact Massif Armoricain-Bassin Parisien entre Angers et Laval (France), Geodinamica Acta, vol. 7, n 3, p. 158-168.

Larue J.-P., Étienne R., 2001. Morphodynamique fluviale et tectonique : l'exemple de la vallée de la Loire dans le sud du Bassin parisien (France), Géomorphologie : relief, processus, environnement, $\mathrm{n}^{\circ}$ 4, p. 281-294.

Lecointre G., 1931. Révision de la feuille de Tours au 1/80 000, Bull. Serv. Carte Géologique France, $n^{\circ} 187$, XXXVI, p. 131-133.
Levant M., Anthore R., Dupont J.-P., Hallégouët B., Robbe D., 1987. Intercomparaison de méthodes microgranulométriques appliquées à des loess. In Pécsi M., French H.M. (eds): Loess and Periglacal Phenomena. Akadémial Klado, Budapest, p. 11-27.

Lisiecki L.-E., Raymo M.-E., 2005. A Pliocene-Pleistocene stack of 57 globally distributed benthic $\mathrm{d}^{18} \mathrm{O}$ records, Paleoceanography, vol. 20, PA1003, doi: 10.1029/2004PA001071.

Lorain J.-M., Denizot G., Lecointre G., Desprez N., 1972. Notice explicative, Carte géologique France (1/50000), feuille Blois (428). Orléans : BRGM, 20 p. Carte géologique par Lorain J.-M., Denizot G., Desprez N., Caudron M., Martins Cl., Hubert M.-F., Louat O. (1972).

Macaire J.-J., 1981. Contribution à l'étude géologique et paléopédologique du Quaternaire dans le SW du Bassin de Paris (Touraine et abords), thèse d'État en Sciences, Tours, $\mathrm{t} 1$ : 304 p., t 2 : 146 p.

Macaire J.-J., 1983. Évolution du réseau hydrographique dans le sud-ouest du Bassin de Paris pendant le Pliocène et le Quaternaire, Bulletin de l'Association française d'étude du Quaternaire, no 4, p. 183-195.

Macaire J.-J., 1984. Les vallées et formations alluviales plioquaternaires dans le $\mathrm{S}$ et le SW du Bassin de Paris : genèse et signification dynamique, Bulletin de l'Association française d'étude du Quaternaire, no 1-2-3, p. 37-40.

Macaire J.-J., Desprez N., 1977. Notice explicative, Carte géologique France (1/50 000), feuille Montrichard ( $\left.n^{\circ} 459\right)$. Orléans, BRGM, 23 p. Carte géologique par Macaire J.-J. (1977).

Manivit J., Martins C., 1974. Carte géologique de France au $1 / 50000$, feuille Château-Renault ( $\mathrm{n}^{\circ} 427$ ), notice explicative 36 p., BRGM, Orléans.

Mason C.C., Folk R.L., 1958. Differentiation of beach, dune and eolian flat environments by size analysis, Mustang Island, Texas, Journal of Sedimentary Petrology, $\mathrm{n}^{\circ} 28$, p. $211-226$.

Mégnien C., Mégnien F., Debrand-Passard S., 1980. Synthèse géologique du Bassin de Paris, mémoire du BRGM, $\mathrm{n}^{\circ} 101,466 \mathrm{p}$.

Miall A.D., 1996. The geology of fluvial deposits: sedimentary facies basin analysis and petroleum geology, Springer Verlag, Berlin, $582 \mathrm{p}$.

Nomade S., Pastre J.-F., Guillou H., Faure M., Guerin C., Delson E., Debard E., Voinchet P., Messager E., 2014 a. ${ }^{40} \mathrm{Ar} /{ }^{39} \mathrm{Ar}$ constraints on some landmark Late Pliocene to Early Pleistocene large mammalian paleofauna: paleoenvironmental and paleoecological implications, Quaternary Geochronology, vol. 21, p. 2-15.

Nomade S., Pastre J.-F., Nehlig P., Guillou H., Scao V., Scaillet S., 2014b. Tephrochonology of the Mont-Dore volcanic Massif (Massif Central, France): new ${ }^{40} \mathrm{Ar} /{ }^{39} \mathrm{Ar}$ constraints on the Late Pliocene and Early Pleistocene activity, Bulletin of Volcanology, vol.76 : 798 DOI 10-1007/ s00445-014-0798-6.

Parfenoff A., Pomerol C., Toureng J., 1970. Les minéraux en grains, méthodes d'étude et détermination, Masson, 578 p. 
Passerat C., 1910. La transgression de la mer des Faluns dans la région de Loire, Annales de Géographie, XIX, p. 350-358.

PAstre J.-F., 1986. Altération et paléoaltération des minéraux lourds des alluvions pliocènes et pléistocènes du bassin de l'Allier (Massif Central, France), Bulletin de l'Association française pour l'étude du Quaternaire, II ${ }^{\mathrm{e}}$ série, vol. 27-28, no 3-4, p. 257-269.

PAstre J.-F., 1987. Les formations plio-quaternaires du Bassin de l'Allier et le volcanisme régional (Massif Central, France), thèse de l'université Paris VI, 706 p.

Pastre J.-F., 2004. The Perrier Plateau : A Plio-Pleistocene long fluvial record in the Allier basin, Massif Central, France, Quaternaire, vol. 15, nº 1-2, p. 87-101.

Pastre J.-F., Cantagrel J.-M., 2001. Téphrostratigraphie du Mont-Dore (Massif Central, France), Quaternaire, vol. 12, $n^{\circ} 4$, p. 249-267.

Rasplus L., 1978. Contribution à l'étude géologique des formations continentales détritiques tertiaires de la Touraine, de la Brenne et de la Sologne, thèse Doctorat Sciences Naturelles, Orléans, 3 vol., 454 p., 25 pl., 13 cartes h.t.

Rasplus L., Alcaydé G., Desprez N., 1974. Carte géologique de France au 1/50000, feuille Tours ( $\left.\mathrm{n}^{\circ} 457\right)$, notice explicative 17 p., BRGM, Orléans.

Tissoux H., Despriée J., Voinchet P., Bahain J.-J., Falguères C., Duvialard J., 2011 . Intérêt de la datation par ESR d'un transect complet pour la compréhension d'un système fluviatile : exemple de la vallée du Loir, Quaternaire, vol. 22, $n^{\circ} 4$, p. 343-355.

Tissoux H., Prognon F., Voinchet P., Lacquement F., TourLiÈre B., Bahain J.-J., 2013. Apport des datations ESR à la connaissance des dépôts sableux plio-pléistocènes en Sologne, premiers résultats, Quaternaire, vol. 24, n 2, p. 141-153.

Tourend J., 1989. Les sables et argiles du Bourbonnais : une formation fluvio-lacustre d'âge pliocène supérieur, étude minéralogique, sédimentologique et stratigraphique, Document du BRGM, no 174, 333 p.

Tourend J., Decaillot P., Pomerol C., 1971. Origine armoricaine des minéraux lourds de la mer des faluns. Mise en doute de la capture de la pré-Loire à l'Helvétien inférieur, Compte Rendu Sommaire de la Société Géologique de France, p. 65-67.

Tourend J., Turland M., 1982. Datation et corrélations de formations détritiques azoïques par les minéraux lourds des volcans du Massif Central. Synchronisme du Tertiaire terminal de Cosne d'Allier et des Sables et Argiles du Bourbonnais à l'aide des zircons volcaniques, Comptes Rendus de l'Académie des Sciences, Paris, t. 294, série II, p. 391-394.

Tourenq J., Pomerol C., 1995. Mise en évidence, par la présence d'augite du Massif central, de l'existence d'une préLoire-pré-Seine coulant vers la Manche au Pléistocène, Comptes Rendus de l'Académie des Sciences, Paris, t. 320, série II a, p. 1163-1169.

TRASK P.D., 1932. Origin and environment of source sediments of petroleum. Gulf Publishing Company, Houston, 67 p.

Vatan A., 1947. La sédimentation continentale tertiaire dans le Bassin de Paris méridional, Thèse Etat Sciences, Paris, Ed. toulousaines de l'ingénieur, 215 p., 7 pl.

Visher G.S., 1969. Grain size distributions and depositional processes, Journal of Sedimentary Petrology, n³9, p. 10741106.

Voinchet P., Despriée J., Gageonnet R., Bahain J.-J., Tissoux H., Falguères C., Dépont J., Dolo J.-M., Courcimault G., 2007. Datation par ESR de quartz fluviatiles dans le bassin de la Loire moyenne en région Centre: mise en évidence de l'importance de la tectonique quaternaire et de son influence sur la géométrie des systèmes de terrasses, Quaternaire, vol. 18, n 4, p. 335-347.

Yvard J.-C., 1968a. Le Miocène continental de la Champeigne tourangelle, Bulletin de Recherches Géologiques et Minières, $2^{\mathrm{e}}$ série, sect. I, n ${ }^{\circ} 1$, p. 5-41.

Yvard J.-C., 1968b. Formations éoliennes des plateaux de la région de Tours, Bulletin de l'Association française d'étude du Quaternaire, no 2, p. 101-110.

Yvard J.-C., 1978. Géomorphologie du sud-onest du Bassin de Paris, Thèse État, Paris IV, 4 vol., 1174 p.

Yvard J.-C., 1995. Sur l'implantation des vallées du bassin moyen de la Loire, Norois, nº 166, p. 381-387. 


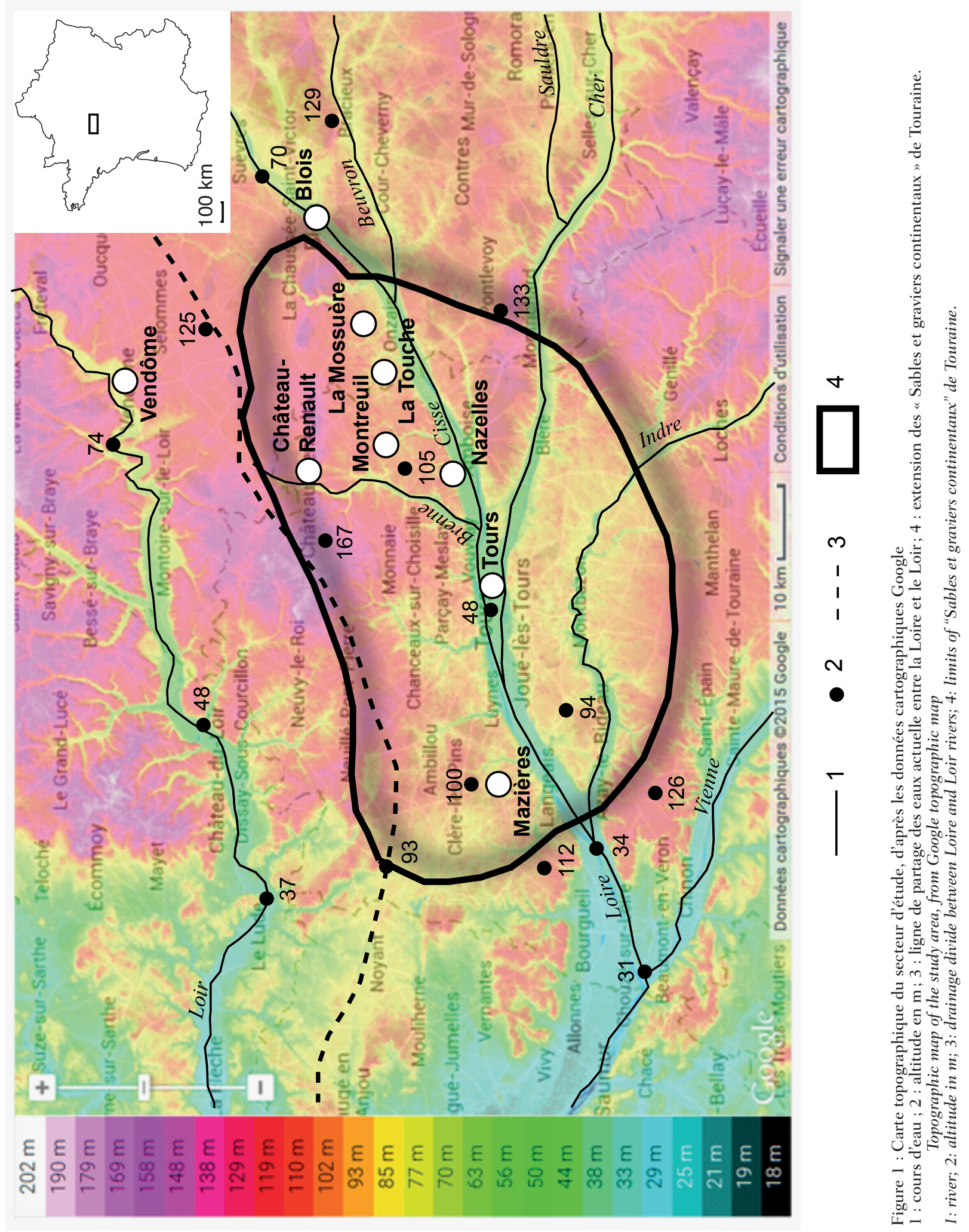

